\title{
Évolutions des organisations scolaires face aux mutations socioéconomiques
}

Le cas de quatre pays d'Asie : la Chine, la Corée du Sud, le Laos, le Sri Lanka

Changes in school education systems as a result of socioeconomic transformations. Case studies: China, South Korea, Laos, Sri Lanka Evoluciones de las organizaciones escolares frente a las mutaciones socioeconómicas. Estudios de casos: China, Corea del Sur, Laos, Sri Lanka

Odile Luginbühl

\section{OpenEdition Journals}

Édition électronique

URL : https://journals.openedition.org/ries/4364

DOI : $10.4000 /$ ries.4364

ISSN : 2261-4265

Éditeur

France Education international

Édition imprimée

Date de publication : 4 avril 2015

Pagination : 119-129

ISBN : 9782854206067

ISSN : 1254-4590

\section{Référence électronique}

Odile Luginbühl, «Évolutions des organisations scolaires face aux mutations socioéconomiques ». Revue internationale d'éducation de Sèvres [En ligne], 68 | avril 2015, mis en ligne le 04 avril 2017, consulté le 02 juillet 2021. URL : http://journals.openedition.org/ries/4364 ; DOI : https://doi.org/ $10.4000 /$ ries. 4364 


\title{
Évolutions des organisations scolaires face aux mutations socioéconomiques
}

\author{
Le cas de quatre pays d'Asie: \\ la Chine, la Corée du Sud, le Laos, le Sri Lanka
}

\author{
Odile Luginbühl \\ Inspectrice d'académie, \\ Inspectrice pédagogique régionale honoraire,
}

France

Mondialisation, pays émergents, croissance du PIB : ces différentes notions géopolitiques dessinent à grands traits les mutations socioéconomiques que connaissent de nombreux pays d'Asie. Ces transformations engendrent de profonds bouleversements dans des pays qui, pour la plupart, sont marqués à la fois par des cultures traditionnelles où coexistent différentes ethnies, langues et religions, et par l'influence de périodes de colonisation, plus ou moins longues et sous diverses formes, soit occidentales, soit internes à la région.

En quelques décennies, ces sociétés complexes ont vécu une transition rapide vers la modernité dans ses formes économiques et technologiques les plus avancées en prenant place dans le jeu international de la mondialisation. Les conséquences de ce choc brutal tendent à accroître les disparités sociales et géographiques, avec une forte urbanisation associée à un exode rural et à des inégalités régionales, mais aussi à développer des besoins et des demandes sociétales qui placent l'éducation et la formation parmi les défis majeurs de ces pays. Comment les systèmes éducatifs évoluent-ils face à ces mutations ? Comment volonté politique et pression de la société s'articulent-elles?

Cet article s'appuie sur les communications présentées dans l'atelier que nous avons coordonné sur ce thème lors du colloque sur l'éducation en Asie ${ }^{1}$ organisé par le Centre international d'études pédagogiques (CIEP), en juin 2014 à Sèvres. Afin d'apporter un éclairage sur les enjeux de l'éducation dans ce contexte historiquement sans précédent, quatre pays situés dans des sphères régionales géographiquement distinctes ont été étudiés : la Chine et la Corée du Sud pour l'Asie de l'Est (Chen, 2014 ; Lee, 2014), le Laos pour l'Asie du Sud-Est (Chounlamany, 2014) et le Sri Lanka pour l'Asie du Sud (Little, 2014)².

\footnotetext{
1. Colloque "L'éducation en Asie en 2014 : quels enjeux mondiaux ? ", organisé les 12, 13 et 14 juin 2014 à l'occasion du $20^{\mathrm{e}}$ anniversaire de la Revue internationale d'éducation de Sèvres. Pour en savoir plus : http://ries.revues. org/3710 (NdIR)

2. Sauf mention contraire, toutes les citations concernant ces pays sont extraites de ces communications publiées sur le site de la revue sur le portail OpenEdition revues.org : http://ries.revues.org/1730 (NdIR)
} 


\section{LES CONTEXTES SOCIOÉCONOMIQUES}

Mettre en parallèle des observations sur plusieurs pays induit, explicitement ou non, des comparaisons. Cette démarche ne peut prendre sens que si chacun des éléments est éclairé par les caractérisations politiques, géographiques, culturelles et économiques qui le constituent et qui vont singulariser les problématiques éducatives nationales.

$\mathrm{Au}$ cours du $\mathrm{XX}^{\mathrm{e}}$ siècle, chacun des quatre pays étudiés a connu des périodes de troubles politiques, tant du fait de la colonisation, en particulier pour le Laos et le Sri Lanka, que des conséquences, directes ou indirectes, des guerres qui ont bouleversé l'Asie après la Seconde Guerre mondiale. Le milieu du $\mathrm{XX}^{\mathrm{e}}$ siècle représente une période-clé de leur évolution : accès à l'indépendance pour le Sri Lanka (1948) et le Laos (1953), mise en place d'un nouveau régime politique, encore aujourd'hui en vigueur, pour la Corée du Sud (1948) et la Chine (1949). Mais ces tournants historiques ont été accompagnés ou suivis de périodes d'instabilité, sous l'effet des guerres de Corée, puis d'Indochine et du Vietnam, qui ont entraîné des guerres civiles, des partitions territoriales et des basculements politiques. Au Sri Lanka, le conflit entre les communautés cingalaise et tamoule, latent depuis plusieurs décennies, se transforma au début des années 1980 en une guerre civile de trente ans, officiellement terminée en 2009.

Malgré les profondes secousses politiques qui les ont traversés, les quatre pays ont en commun de s'inscrire depuis une période récente dans une dynamique de fort développement économique national : en 2012, la croissance du PIB était de 8,2 \% pour le Laos, 7,8 \% pour la Chine (classée première puissance économique du monde en $2014^{3}$ ) et $6,4 \%$ pour le Sri Lanka (Brun, 2014) ${ }^{4}$. Seule la Corée du Sud, dont la progression a commencé plus tôt, présentait une croissance de $2 \%{ }^{5}$.

Mais cet essor économique spectaculaire se produit dans des conditions très différentes pour chaque pays, tout d'abord en raison d'un décalage temporel dans son émergence. La Corée du Sud, un des quatre "dragons asiatiques " ${ }^{6}$, est entrée dans une période de croissance économique depuis la fin des années 1950, alors que la Chine ne s'est ouverte à la mondialisation que dans les années 1980, comme le Sri Lanka (mais celui-ci dans un contexte de guerre civile), et le Laos au début des années 1990. La plus ou moins grande ancienneté de la prospérité économique des pays se traduit dans leurs capacités d’investissement dans le

3. Source : Fonds monétaire international, World Economic Outlook Database [en ligne] http://goo.gl/sCBcKy/

4. Sauf mention contraire, les données statistiques figurant dans cet article proviennent du document élaboré pour le colloque par Céline Brun, du centre de ressources et d'ingénierie documentaires du CIEP. Les chiffres fournis correspondent à des données établies en 2012 et 2013 (Brun, 2014).

5. Pour la même année 2012, la moyenne des pays de l’Union européenne affichait un taux de - 0,4 \% (données INSEE, source : Eurostat).

6. Avec Singapour, Hong-Kong et Taïwan. 
secteur de l'éducation (5,25\% du PIB en Corée du Sud contre 2,8 \% au Laos et $1,7 \%$ au Sri Lanka), auxquelles s'ajoute une plus ou moins grande participation de l'aide internationale.

La démographie des quatre pays est très contrastée, en nombre total comme en répartition par catégories d'âge. Aux extrêmes se situent la Chine, avec 1,3 milliard d'habitants, et le Laos, qui en compte moins de 7 millions; dans une position intermédiaire, la Corée du Sud (50 millions d'habitants) et le Sri Lanka (21 millions). Le Laos et le Sri Lanka sont des pays « jeunes », avec respectivement $36 \%$ et $26 \%$ de leur population âgée de moins de 14 ans. La Chine et la Corée du Sud, avec $16 \%$ de leur population dans cette tranche d'âge, présentent un profil plus proche des moyennes européennes. Les stratégies en matière d'éducation vont donc différer en fonction des données démographiques : les enjeux porteront en priorité sur l'enseignement de base ou sur les formations secondaires et supérieures, selon la part de la population concernée.

À ces écarts démographiques entre les quatre pays s'ajoute, à l'intérieur de chacun d'eux, une diversité ethnique et linguistique particulièrement marquée en Chine, au Laos et au Sri Lanka. La Corée du Sud se distingue de ce groupe par son homogénéité : la proportion d'immigrants, bien qu'en progression, reste faible et le coréen est la seule langue officielle. Cas extrême, la population du Laos - 6,8 millions d'habitants - comprend 49 ethnies qui parlent 82 langues. Le laotien, langue officielle et unique langue d'enseignement, n'est la langue maternelle que d'environ la moitié de la population (le groupe des Lao Loum) - ce qui n'est pas sans conséquences sur les conditions d'apprentissage scolaire: l'aisance linguistique, associée aux représentations traditionnelles des groupes ethniques et des relations garçons/filles font que « les enseignants, plus ou moins consciemment, établissent des hiérarchies qui placent les élèves garçons d'origine Lao Loum au sommet ». En Chine, si $92 \%$ de la population appartiennent au groupe ethnique des Han, 55 autres ethnies, appelées "nationalités », sont officiellement reconnues; le mandarin est la langue officielle nationale, mais la plupart de ces ethnies, qui regroupent environ 120 millions de personnes, possèdent leur propre langue. Au Sri Lanka, le groupe ethnique cinghalais représente $72 \%$ de la population et le groupe des Tamouls (Tamouls indiens et Tamouls cinghalais) $15 \%$, le reste de la population se partageant entre plusieurs ethnies minoritaires (Government of Sri Lanka, 2012). La question linguistique y est un enjeu majeur : l'usage de la seule langue cinghalaise en tant que langue officielle au détriment du tamoul a été perçu comme une manifestation de discrimination ethnique qui alimenta le conflit entre les communautés et conduisit à la guerre civile. Les deux langues ont maintenant le statut de langue officielle et de langue d'enseignement. Un tel foisonnement linguistique, dans les trois pays concernés, constitue non seulement un enjeu pour les systèmes éducatifs en termes de cohésion sociale, mais aussi une donnée essentielle à prendre en compte pour assurer un accès équitable de tous à l'éducation - ces deux objectifs étant énoncés dans les textes officiels de référence de ces pays. 
Autre aspect des disparités sociétales : les inégalités territoriales, par exemple entre la Chine de l'est (littorale), très urbanisée avec une forte densité de population, et la Chine de l'intérieur, où la population se répartit sur un territoire beaucoup plus vaste. De manière générale, le clivage villes/campagne joue un rôle déterminant dans le développement de l'éducation en raison des problématiques spécifiques de la scolarisation dans les territoires ruraux, et notamment par rapport au principe d'équité déjà évoqué. Les populations rurales sont pauvres pour la plupart, ce qu'indique la comparaison entre leur part démographique dans la population totale et la part du secteur agricole dans le PIB national.

Ici encore, la Corée du Sud connaît une situation distincte de celle des trois autres pays avec une dominante de population urbaine et une faible proportion de ruraux (17\%), pour une part de l'agriculture dans le PIB de 2,6 \%. Au Sri Lanka, en revanche, le poids démographique des campagnes est considérable puisqu'il atteint $85 \%$ de l'ensemble de la population, bien que la part de l'agriculture dans le PIB ne soit que de 10,6 \%. Malgré leur nombre, les jeunes ruraux ne disposent que d'une offre de formation restreinte:

L'accès aux sciences, à l'informatique et à l'anglais ainsi qu'aux opportunités post-secondaires du secteur privé qui ouvrent la voie à l'emploi dans l'économie libéralisée se concentre dans les milieux urbains.

$\mathrm{Au}$ Laos, la population rurale est également importante $(65 \%)$, pour une part du PIB plus élevée (24,8\%). La scolarisation dans le primaire y est assurée dans l'ensemble, mais beaucoup plus discriminante dans le secondaire :

Alors que 30,6 \% des jeunes urbains Lao-Tai non-pauvres ont accès au premier cycle de l'enseignement secondaire, seulement $3 \%$ des jeunes ruraux, non Lao-Tai et pauvres y ont accès. De plus, les populations urbaines ont accès à des écoles mieux équipées que celles des territoires ruraux. (Unesco, 2011).

En Chine, la répartition entre villes et campagne, apparemment presque équivalente ( $48 \%$ de ruraux), recouvre une réalité économique beaucoup plus contrastée puisque l'agriculture ne produit que 9,7 \% du PIB. Ce dernier indicateur explique l'importance des flux migratoires internes en Chine, des campagnes vers les villes et de l'ouest du pays vers la côte est, ce qui pose un problème aigu d'accueil scolaire pour les enfants des migrants ruraux dans des villes pléthoriques, où « l'urbanisation continue à augmenter à un rythme moyen de $2 \%$ par an ».

\section{ÉDUCATION ET MUTATIONS SOCIOÉCONOMIQUES}

Comment ces sociétés-mosaïques répondent-elles à l'explosion des besoins d'éducation et de formation qu'entraînent leur croissance démographique et leur essor économique spectaculaire? Question d'autant plus complexe 
que la culture scolaire traditionnelle imprègne encore fortement les systèmes éducatifs. De façon assez convergente dans les pays étudiés, cette culture traditionnelle est caractérisée par trois éléments principaux :

- la représentation de l'éducation dans la société, comme valeur morale sous l'influence de la culture confucéenne de la méritocratie, mais aussi comme facteur d'ascension sociale : en Chine, " la réussite des études inspire le respect » et en Corée du Sud, pays où le taux de mobilité sociale est élevé, "se consacrer à ses études est une attitude fortement valorisée dans la société et encouragée par la famille »;

- un enseignement orienté par et pour la réussite aux examens : en Chine, l'éducation est encore fortement marquée par le système des concours impériaux, selon lequel «le résultat au concours est plus important que les connaissances et la compétence » et " étudier revient à apprendre les techniques pour réussir le concours »; l'enjeu de promotion sociale attaché autrefois aux concours impériaux est conféré maintenant au concours d'entrée à l'université, le gaokao, avec des conséquences semblables sur les objectifs et modalités de l'enseignement; de même, en Corée, « la focalisation de l'enseignement et de l'apprentissage sur la compétition à l'examen » est un héritage de la culture scolaire traditionnelle, qui soulève néanmoins des critiques dans le pays; cette centration sur la compétition est un des principaux facteurs du développement des cours privés en complément de l'enseignement public ;

- des démarches pédagogiques fondées sur les cours magistraux et la mémorisation par les élèves qui laissent peu de place à leurs réflexion et créativité personnelles : «la mémorisation est un facteur de réussite primordial [...], la mémorisation exigeant acharnement et persévérance, les études sont par définition difficiles et sans plaisir» (Chine) ; au Laos, "les enseignants demeurent des figures d'autorité [...], les élèves copient ce que leur dicte le maître et apprennent par cour» (Laos).

$\mathrm{Si}$, dans chacun des quatre pays, on observe une tension entre ces modèles traditionnels d'éducation et l'indispensable adaptation des systèmes éducatifs aux transformations économiques et sociétales, elle se manifeste différemment selon les contextes nationaux. C'est en Corée du Sud, pays dont les scores aux enquêtes PISA et TIMSS sont des plus performants, que la relation entre la réussite économique et les réformes du système éducatif semble la plus étroite :

L'augmentation des taux de scolarisation, la réforme des systèmes d'enseignement, l'élévation du niveau d'exigence dans le curriculum national et les manuels scolaires, l'amélioration de la qualité des enseignants et l'importance des enjeux des examens, sont autant de facteurs qui concourent à l'efficacité du système. Ils ont par ailleurs contribué à assurer la remarquable croissance économique du pays et le développement de la démocratie sociale durant les six décennies écoulées. 
Dans les trois autres pays, l'évolution du système éducatif s'est effectuée plus difficilement et demeure insuffisante aux yeux des élèves et des familles. Au Sri Lanka, un décalage se manifeste entre la croissance du pays et l'accès à l'éducation et à la formation professionnelle : " les attentes des jeunes en matière d'éducation et d'emploi sont bien plus élevées qu'avant, mais pour beaucoup de jeunes ces attentes restent insatisfaites ", malgré une augmentation du taux de scolarisation. L'ouverture économique du pays, à partir du début des années 1980, a creusé des écarts dans la population :

[...] l'accès aux diplômes, qu'ils soient étrangers ou nationaux, s'est accru dans tous les groupes sociaux, mais les groupes les plus aisés ont un meilleur accès aux examens étrangers qui sont considérés comme plus rentables sur le marché du travail.

Dans le cas du Laos, les modifications en matière d'éducation résultent d'une volonté politique qui peine à être mise en œuvre dans les faits. L'évolution du système a été impulsée de l'extérieur, parallèlement à l'ouverture du pays à des marchés internationaux, sous l'influence de bailleurs de fonds dont l'aide financière à l'éducation était soumise à un assouplissement du mode de gestion du système vers plus de décentralisation et à des innovations dans les démarches pédagogiques. Mais les réformes mises en place à partir de 1994 pour répondre à ces injonctions se heurtent, dans la pratique, à des freins structurels (rigidité d'un système centralisé et hiérarchisé) et culturels (poids des traditions d'enseignement, questions liées à l'appartenance ethnique et à l'égalité des sexes). La volonté institutionnelle de changement ne peut devenir effective par de seuls décrets "descendants » et, confrontée aux pratiques et représentations locales, elle est à l'origine de "situations hybrides », symptomatiques du bouleversement du pays et de la façon dont « les forces locales répondent aux forces mondiales ».

En Chine, si l'enseignement primaire est favorablement apprécié, les critiques portent sur les enseignements secondaire et supérieur, écartelés entre tradition et mondialisation :

Sous l'ombre de la tradition et du gaokao, l'éducation scolaire ressemble de plus en plus à une super machine de préparation aux concours et s'adapte de moins en moins à l'énorme mutation que connaît actuellement la société chinoise.

Cette inadéquation de la formation des jeunes aux demandes sociétales conduit un nombre important d'étudiants chinois à poursuivre leurs études supérieures à l'étranger :

La croissance explosive du nombre d'étudiants chinois partant à l'étranger s'explique par beaucoup de raisons, en particulier par l'insatisfaction, voire la déception, à l'égard du système scolaire actuel trop orienté vers le concours.

Là encore, des réformes sont en œuvre, avec des expérimentations menées à Shanghai, "ville la plus ouverte et la plus internationalisée de la Chine » et véritable laboratoire de l'éducation en Chine. 


\section{DÉBATS ET ÉVOLUTIONS ACTUELLES}

On observe donc dans les quatre pays une constante, et souvent difficile, recherche d'ajustement des politiques éducatives aux transformations qui bousculent les systèmes traditionnels. Quelles en sont les priorités actuelles, après trente à soixante ans de croissance économique (selon les pays) et, par conséquent, de confrontations aux mutations de la société ? Deux objectifs principaux, communs aux quatre systèmes éducatifs - au moins dans leur visée -, orientent leurs évolutions : assurer l'équité dans l'accès à l'éducation, c'est-à-dire remédier aux disparités (géographiques, ethniques ou entre les sexes), et améliorer la qualité de l'enseignement, c'est-à-dire adapter contenus et méthodes aux attentes de la société. Ces deux objectifs sont, dans une certaine mesure, interdépendants car les modifications dans les apprentissages et les démarches pédagogiques, qui visent à mieux préparer les jeunes aux changements du monde contemporain, peuvent également contribuer à assurer une meilleure prise en compte des spécificités des élèves.

\section{Améliorer la qualité de l'enseignement}

Même en Corée du Sud, malgré les scores élevés des élèves aux enquêtes internationales, des dispositifs visant à conforter la qualité et l'efficacité du système ont été récemment mis en œuvre. En s'appuyant sur les résultats aux évaluations nationales, une politique de soutien aux élèves en difficulté a été mise en place dans le cadre d'un réseau d' "écoles du progrès » qui disposent de moyens supplémentaires, tant sur le plan administratif et financier que dans le domaine de la formation des enseignants, avec pour contrepartie une évaluation de leurs résultats. L'implication des familles est également encouragée. Le fonctionnement de ce dispositif a fait l'objet d'une analyse favorable, confirmée par le fait qu'un premier bilan a permis de diminuer le nombre d'établissements concernés, passant de 1440 en 2009 à 650 en 2012. Il faut cependant souligner que l'évaluation porte sur "la compétence cognitive des élèves, évaluée selon les critères d'examens à enjeux élevés ", sans prendre en compte d'autres aspects de l'éducation, tels que le développement de la personne. Cette problématique a été mise en lumière au niveau national, notamment par l'analyse des résultats de l'enquête PISA 2012, qui place la Corée du Sud parmi les meilleurs sur 65 pays (cinquième rang pour les mathématiques, deuxième rang pour la résolution de problèmes), mais au dernier rang pour le "pourcentage d'élèves déclarant se sentir bien à l'école» (OCDE, 2014a).

L'évolution vers un enseignement et une pédagogie impliquant les élèves dans les apprentissages est une préoccupation commune au Laos et à la Chine. Au Laos, cette démarche est entreprise depuis quelques années, notamment avec la possibilité de consacrer $20 \%$ du programme à des adaptations au contexte 
local, permettant ainsi de "favoriser la participation active et les méthodes de découverte ». Mais peu d'enseignants se saisissent de cette offre par manque de formation à l'élaboration de projets éducatifs et de supports pédagogiques pour développer un travail autonome. En 2009, un rapport d'évaluation du ministère de l'éducation a reconnu « les difficultés qu'il y a eu à mettre en place de nouvelles méthodes d'enseignement en raison des compétences insuffisantes des enseignants, de programmes peu appropriés ou encore du manque de matériels pédagogiques ». Une volonté politique de changement s'exprime dans le Plan national de réforme du système éducatif pour la période 2006-2015, qui prévoit la mise en place d'un nouveau curriculum intégré pour les cycles correspondant à l'éducation de base (pré-primaire, primaire et premier cycle du secondaire), dont les contenus et les niveaux attendus devraient s'accorder aux standards internationaux (Ministry of Education, 2008). Parallèlement, l'accent est mis sur le programme de formation des maîtres qui doit être repensé en fonction du nouveau curriculum.

En Chine, c'est à Shanghai que l'expérimentation d'une "éducation nouvelle visant le développement intégral des élèves " a été entreprise dès les années 1980. La même volonté qu'au Laos de transformer les méthodes d'apprentissage traditionnelles a conduit à une redéfinition du cursus en champs disciplinaires reconstruits autour de grands pôles, avec une part de travail autonome et une organisation des cours qui sollicite davantage la participation des élèves. Même si ces démarches innovantes sont encore limitées par l'enjeu des examens, elles n'en constituent pas moins une transformation considérable, qui « implique une véritable subversion de l'autorité de l'enseignant qui, selon l'idée traditionnelle et orthodoxe, détient la vérité absolue et a donc toujours raison». Sur un plan plus structurel, la politique éducative menée à Shanghai a consisté à réduire les différences entre "écoles faibles " et "écoles fortes " par des mesures de soutien spécifique pour les premières (augmentation du budget, formation des enseignants), mais aussi par un dispositif d' «alliances scolaires » ou jumelages qui créent un réseau pédagogique entre "fortes » et "faibles ». Autre évolution considérable : l'allégement des contraintes liées aux seuils que représentaient les concours, tant pour l'entrée au collège (suppression du concours, d'où plus de place accordée à l'innovation dans les écoles primaires) qu'à l'université (où le rôle du gaokao a été minoré et ses modalités assouplies). Les résultats positifs de ces mesures sur l'amélioration de la qualité de l'enseignement ont incité le ministère de l'éducation nationale à en reprendre les principes dans le cadre du Programme national à long et moyen terme sur la réforme et le développement du système éducatif (2010-2020), confirmant ainsi que «Shanghai représente la tendance de l'évolution en Chine». 


\section{Assurer l'équité dans l'accès à l'éducation}

Si l'amélioration de la qualité de l'enseignement contribue à la réduction des inégalités, il n'en demeure pas moins indispensable de développer des actions spécifiques pour des groupes d'élèves dont l'accès à l'éducation est rendu plus difficile en raison des disparités sociales déjà évoquées.

L'accès de tous les enfants à l'éducation est un principe inscrit dans les quatre systèmes éducatifs observés. Au niveau de l'enseignement primaire, les données statistiques indiquent de nets progrès dans les taux de scolarisation mais d'importants écarts subsistent, notamment au Laos, au Sri Lanka et en Chine, en fonction de trois paramètres déjà évoqués : la situation géographique (villes/ campagne), l'appartenance ethnique et le genre (garçons/filles). Ces facteurs d'exclusion pèsent encore plus lourdement pour l'accès à l'enseignement secondaire.

[Ainsi, au Laos], les jeunes issus de familles pauvres et nombreuses, appartenant à une minorité ethnique et vivant dans des zones rurales et isolées, n'ont aucune possibilité de poursuivre leurs études dans l'enseignement secondaire. C'est en particulier le cas des filles qui vivent loin de l'école et abandonnent leurs études pour aider leurs parents à travailler dans les rizières ou s'occuper de la maison.

Cette forme de ségrégation éducative se manifeste aussi au Sri Lanka :

[Des] disparités importantes subsistent en fonction du type d'école, de la langue d'enseignement, de l'emplacement de l'établissement ainsi qu'entre les garçons et les filles.

Les différentes réformes mises en place ces dernières années dans chaque pays affichent l'objectif de l'accès de tous à l'éducation, mais il reste à le rendre pleinement effectif et à l'étendre aux différents niveaux d'enseignement.

La question de la scolarisation des enfants de migrants se pose particulièrement en Chine et en Corée, bien qu'il s'agisse de types de migrations différents. En Chine, les ruraux qui viennent travailler dans les villes sont des migrants de l'intérieur, en nombre de plus en plus important. Leurs enfants d'âge scolaire représentent $20 \%$ des élèves, la majorité d'entre eux (évaluée à 20 millions) vivant en ville avec leurs parents. Leur intégration scolaire soulève deux difficultés : un rejet social, les habitants des villes refusant qu'ils fréquentent les mêmes établissements que leurs enfants, et un problème de financement de leur scolarité, que les villes refusent de prendre en charge si les parents ne disposent pas d'un livret de résidence (hukou) urbain. Ici encore, le modèle shanghaïen a joué un rôle moteur : forte d'une culture de l'ouverture, la ville a financé d'emblée la scolarisation des enfants de migrants en créant des écoles spécifiques, puis, cette discrimination se révélant négative, a modifié sa politique : " aujourd'hui, les enfants de migrants sont tous intégrés dans les écoles publiques et travaillent avec les enfants autochtones ». En 2002, l'État chinois a repris et 
imposé le principe du financement de la scolarisation des enfants de migrants par les villes d'accueil dans les écoles publiques, en faisant prévaloir la mixité sociale, ce qui conduit à une disparition progressive des écoles spécifiques. De plus, le système des livrets de résidence distinctifs pour les urbains et les ruraux devrait disparaître prochainement, ce qui accordera les mêmes droits aux enfants de migrants qu'aux habitants des villes.

En Corée, l'immigration, essentiellement externe au pays, est encore limitée (1,6\% de la population totale en 2011(OCDE, 2014b), mais en augmentation constante en raison de l'attractivité économique du pays et de son évolution démographique (taux de natalité en baisse, vieillissement de la population). Les évaluations nationales ont montré que les enfants issus de cette population multiculturelle rencontrent des difficultés scolaires importantes, avec un taux de décrochage de $43 \%$. Pour remédier à cette situation, une politique éducative a été mise en place à la fin des années 2000, définie par la prise en charge spécifique de ces élèves dans des établissements spécialisés qui dispensent des programmes d'enseignement bilingues/biculturels. Cependant, ces choix sont actuellement discutés car ils tendent à donner une image négative de la population multiculturelle et à rendre plus difficile son intégration au sein d'une société dont « les traditions et la longue histoire maintiennent encore une identité homogène forte » (Lee, 2014). La scolarisation des enfants de migrants constitue une problématique relativement récente en Corée, mais elle est considérée comme un des enjeux des années à venir pour le système éducatif coréen et les débats récents ont mis en évidence la nécessité de " réexaminer les concepts fondamentaux, les objectifs finaux ainsi que les processus de mise en œuvre de l'enseignement multiculturel ».

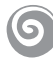

Cette approche des évolutions de quatre systèmes éducatifs confrontés à d'importantes et parfois brutales mutations socioéconomiques fait apparaître, dans le cadre d'une problématique commune de tension entre tradition et adaptation, de nombreuses différences dues à la spécificité des contextes nationaux. Le décalage temporel des phases de développement économique entre les quatre pays joue un rôle important dans le décalage des avancées en matière d'éducation. Si la Corée du Sud s'attache principalement à l'amélioration de la qualité de l'enseignement, le Laos doit encore donner la priorité à l'aspect quantitatif, c'està-dire assurer la scolarisation complète des enfants pour l'enseignement de base.

On observe cependant une même tendance, même si là encore un écart se manifeste, au développement d'outils de pilotage des systèmes, notamment sous forme d'évaluations nationales, qui visent à aider à la mise en place des réformes et à l'ajustement progressif des mesures prises. La recherche en éducation, au sein d'institutions nationales, ministères ou universités, joue 
également un rôle croissant dans la réflexion sur les politiques éducatives avec une perspective d'échanges et de coopération internationale dont le colloque du CIEP fut une manifestation concrète.

\section{BIBLIOGRAPHIE}

BRUN C. (2014) : Sélection de données statistiques, colloque: "L'éducation en Asie en 2014 : quels enjeux mondiaux? ", CIEP, 12-14 juin 2014. http://goo.gl/GnlFMf

CHEN W. (2014) : "Évolutions des organisations scolaires chinoises face aux mutations sociales", Revue internationale d'éducation de Sèvres [en ligne], colloque "L'éducation en Asie en 2014 : Quels enjeux mondiaux? ", mis en ligne le 11 juin 2014. http://ries.revues.org/3755

CHOUNLAMANY K. (2014) : «School education reform in Lao PDR: good intentions and tensions? ", Revue internationale d'éducation de Sèvres [en ligne], colloque "L'éducation en Asie en 2014 : Quels enjeux mondiaux?", mis en ligne le 11 juin 2014. http://ries.revues.org/3750 ; version anglaise : http://ries.revues.org/3766

GOVERNMENT OF SRI LANKA (2012) : Census 2012, Department of census and statistics. http://goo.gl/aWahcN/

LEE H.-W. (2014) : «Deux projets éducatifs en Corée du Sud : les Écoles du progrès et l'Enseignement multiculturel ", Revue internationale d'éducation de Sèvres [en ligne], colloque "L'éducation en Asie en 2014 : Quels enjeux mondiaux ?", mis en ligne le 11 juin 2014. http://ries.revues.org/3764 ; version anglaise : http://ries.revues.org/3897

LITTLE A. (2014) : "Mondialisation et éducation au Sri Lanka ", Revue internationale d'éducation de Sèvres [en ligne], colloque "L'éducation en Asie en 2014 : Quels enjeux mondiaux?", mis en ligne le 11 juin 2014. http://ries.revues.org/3838; version anglaise : http://ries.revues.org/3774

OCDE (2014a) : Principaux résultats de l'enquête PISA 2012. Ce que les élèves de 15 ans savent et ce qu'ils peuvent faire avec ce qu'ils savent, OCDE, 2014. http://goo.gl/P2S3Y9/

OCDE (2014b) : «Population immigrée et étrangère » in Panorama des statistiques de l'OCDE 2014-Economie, environnement et société, OCDE.

MINISTRY OF EDUCATION OF LAOS (2008) : National Education System Reform Strategy 2006-2015, Gouvernement du Laos, avril 2008. http://goo.gl/zAlIsm/

UNESCO (2011) : Données mondiales de l'éducation, 7 édition 2010/11, Bureau international de l'éducation. http://goo.gl/JpxuW7/ 
